Please do not remove this page

RMIT

UNIVERSITY

\title{
Continuous Growth Synthesis of Zinc Oxide Nanocrystals with Tunable Size and Doping
}

Wainer, Pierce; Kendall, Owen; Lamb, Alexander; Barrow, Steven; Tricoli, Antonio; Gomez, Daniel; van Embden, Joel

https://researchrepository.rmit.edu.au/esploro/outputs/9921971673401341/filesAndLinks?institution=61RMIT_INST\&index=null

Wainer, P., Kendall, O., Lamb, A., Barrow, S., Tricoli, A., Gomez, D., van Embden, J., \& Della Gaspera, E. (2019). Continuous Growth Synthesis of Zinc Oxide Nanocrystals with Tunable Size and Doping. Chemistry of Materials, 31, 9604-9613. https://doi.org/10.1021/acs.chemmater.9b02655

Document Version: Accepted Manuscript

Published Version: https://doi.org/10.1021/acs.chemmater.9b02655

Repository homepage: https://researchrepository.rmit.edu.au

(c) 2019 American Chemical Society.

Downloaded On 2023/04/26 12:01:08 +1000 


\section{Continuous Growth Synthesis of Zinc Oxide}

\section{Nanocrystals with Tunable Size and Doping}

Pierce Wainer ${ }^{1}$, Owen Kendall ${ }^{1}$, Alexander Lamb ${ }^{1}$, Steven J. Barrow ${ }^{1}$, Antonio Tricoli ${ }^{2}$, Daniel E. Gomez ${ }^{1}$, Joel van Embden ${ }^{1}$,Enrico Della Gaspera ${ }^{1, *}$

${ }^{1}$ School of Science, RMIT University, Melbourne VIC 3000, Australia.

${ }^{2}$ Nanotechnology Research Laboratory, College of Engineering and Computer Science, The Australian National University, Canberra ACT 2601, Australia. 


\section{ABSTRACT}

Chemical syntheses of nanocrystals using colloidal techniques enable the production of a large variety of nanomaterials of a desired size, shape and composition. Therefore, these syntheses hold tremendous promise for the production of nanoparticles for next-generation technologies. However, they suffer from scalability issues, which can limit, or prevent, their translation into commercial technologies. Here we show the synthesis of zinc oxide nanocrystals with controlled size and doping using a continuous growth method, which is easily scalable. We demonstrate the tunable growth of pure $\mathrm{ZnO}$ nanocrystals from $\sim 5 \mathrm{~nm}$ up to $\sim 30 \mathrm{~nm}$, and the synthesis of plasmonic $\mathrm{ZnO}$ nanocrystals by incorporating substitutional trivalent dopants such as aluminium, gallium and indium. We investigated the growth kinetics of these nanocrystals and used a variety of characterization techniques to fully elucidate the relationship between synthetic conditions and nanocrystal properties. We validate our reaction method by synthesizing Al-doped $\mathrm{ZnO}$ nanocrystals on a gram-scale, and with a reaction yield of $100 \%$. These nanocrystals are used to deposit thin coating with excellent transparency and enhanced electrical conductivity compared to native $\mathrm{ZnO}$. 


\section{INTRODUCTION}

The synthesis of nanocrystals (NCs) using colloidal techniques is one of the more versatile and powerful methods for the preparation of high-quality, stable suspensions of NCs. Obtaining a colloidally stable dispersion of NCs, rather than having clusters of aggregated particles is vital for many applications, including depositing coatings of optical quality, and embedding NCs within matrices. ${ }^{1,2,3}$ Therefore, colloidal reactions for NCs have been constantly refined and improved since the seminal works conducted decades ago. ${ }^{4,5}$ A clear understanding of the nucleation and growth events in colloidal NC syntheses has made possible to create a large library of synthetic methods for the preparation of colloidal NCs, including metals, semiconductors, quantum dots (QDs), oxides, and dielectrics. ${ }^{6,7,8,9,10}$ Among these, doped metal oxides have attracted increasing interest, due to the thermal and chemical stability of metal oxides, as well as the enhanced optical, electrical and magnetic properties that may be imparted to the NCs when appropriately doped. ${ }^{11,12,13,14,15}$ One of the main area of research for doped metal oxides is the synthesis of degenerately (aliovalently) doped wide band gap oxides, for use as transparent electrodes in optoelectronic devices including photovoltaics, light emitting diodes (LEDs) and electrochromics, but also as IR active materials for catalysis and sensing. ${ }^{16,17,18,19}$

The colloidal synthesis of doped metal oxide NCs has been traditionally achieved using hot injection methods, which rely on the swift injection of one or more precursors into a solution containing hot solvents and/or surface ligands to trigger the nucleation of NCs. Despite the high quality of NCs produced, the inherent non-scalable nature of this hot injection event has motivated the search for other types of colloidal synthesis approaches such as heat-up and flow reactions. ${ }^{8,20}$ One of the main methods that has been developed for the synthesis of metal oxide colloidal NCs is the reaction of a metal precursor with water that is formed in-situ from 
an esterification reaction between metal carboxylates and alcohols, at temperatures below those required for the thermal decomposition of the metal carboxylates themselves. This reaction can be used in both hot-injection and heat up protocols, and has been explored for a variety of pure and doped metal oxides including $\mathrm{ZnO}, \mathrm{TiO}_{2}, \mathrm{MnO}$, etc. ${ }^{21,22,23,24}$ However, despite the robustness and versatility of this synthetic protocol, and its proven application within heat-up (potentially scalable) reactions, there is an intrinsic issue with this type of synthesis routes, that hinders their scalability: the amount of water that is generated by the esterification reaction is directly related to the amount of precursors used. If the reaction is scaled up, the excess water generated causes well-known problems such as inhomogeneous nucleation of particles, sudden growth, and precipitation of large aggregates.

A potential solution to this issue has been recently reported by the Hutchison group, which proposed a slow injection approach coupled to a continuous flow of nitrogen gas. ${ }^{25} \mathrm{In}$ this system, metal oleates are slowly injected into a hot solution of oleyl alcohol, causing the resultant esterification reaction to trigger the formation of metal oxide NCs. The excess water that is formed is removed as water vapor thanks to a constant stream of nitrogen flushed through the headspace of the reaction vessel. In conjunction with metal precursors with the right reactivity, this technique enables the highly controlled growth of NCs in a layer-by-layer fashion, similarly of what happens in the production of polymers through living polymerization. ${ }^{26}$

Based on these encouraging results, more work was done on refining this synthetic method to achieve unprecedented control of NC size, dopant concentration and even dopant location in the case of indium oxide. ${ }^{27,28}$ Recently, iron oxide NCs have also been prepared with good control on their size and size distribution using a continuous growth method. ${ }^{29}$ However, adapting this reaction method to other metal oxides has proven highly challenging 
especially in the case of zinc oxide. The synthesis of $\mathrm{ZnO}$ NCs using this method has shown poor reproducibility and low reaction yields. ${ }^{25}$ Moreover, zinc oleate is a solid at room temperature, and therefore the reaction must be done by slowly injecting a hot precursor (above $\sim 80{ }^{\circ} \mathrm{C}$ ) over the course of several minutes, which is problematic and itself becomes a source of irreproducibility. It is worth highlighting at this stage that $\mathrm{ZnO}$ colloidal NCs have been widely investigated for decades, starting from the seminal works of Bahnemann and Spanhel involving the precipitation of $\mathrm{ZnO}$ clusters in alcohols using bases such as $\mathrm{NaOH}$ and $\mathrm{LiOH}^{30,31}$ Several reaction methods for $\mathrm{ZnO}$ have been developed since, including forced hydrolysis, solvothermal reactions, and high temperature colloidal reactions. ${ }^{32,33,34,35,36,37}$ Of all the available methods, high temperature reactions (either through heat up or hot injection) provided the best results in terms of NC quality, colloidal stability, and tunability of size, shape and composition (especially aliovalent doping). However, the issues associated with scalability (such as the in-situ water generation mentioned earlier) still plague these reactions, and therefore a synthesis that enables control of NC properties using a scalable protocol is highly desirable.

In this study we overcome all these issues by developing a synthesis for $\mathrm{ZnO} \mathrm{NC}$ based on a slow injection approach, through the use of a zinc precursor that is not only liquid at room temperature, but is also more reactive. This drastically increases the reproducibility of our slow injection process, and also results in a reaction yield close to $100 \%$. We successfully developed a robust reaction protocol that enables the synthesis of $\mathrm{ZnO}$ NCs with fine control over their size and doping levels. Our reaction protocol is also readily scalable and enables the isolation of grams of materials without any of the aforementioned issues.

\section{RESULTS AND DISCUSSION}


We firstly formed zinc oleate by reacting zinc acetate with oleic acid at $\sim 100{ }^{\circ} \mathrm{C}$ under vacuum, in order to remove the acetic acid formed in the process. Preliminary reactions were carried out through either the injection of hot $\left(\sim 80^{\circ} \mathrm{C}\right)$ zinc oleate, or through the injection of commercially available zinc neodecanoate (a zinc carboxylate which is liquid, albeit very viscous, at room temperature) into hot oleyl alcohol. However, these reactions did not produce any appreciable recoverable material, suggesting a low reactivity of zinc carboxylates alone. We therefore decided to modify the zinc oleate to achieve the dual goal of increasing its reactivity towards the formation of $\mathrm{ZnO}$ via the esterification with oleyl alcohol, and of increasing its stability at low temperatures, enabling a simpler and more reproducible synthetic protocol. Once the metal oleate complex was formed as mentioned earlier, oleylamine was added. Amines are commonly used to form complexes with zinc, ${ }^{38}$ and in fact this is one of the main strategies used to solubilize zinc acetate in alcohols for use as sol-gel precursors for $\mathrm{ZnO} .{ }^{39}$ Through this strategy, we prepared a zinc precursor which can be cooled down to room temperature obtaining a viscous liquid, suitable for a continuous slow injection over the course of several minutes. In addition, the prepared precursor enabled the nucleation and growth of $\mathrm{ZnO} \mathrm{NCs}$ very reproducibly within a range of reaction temperatures, with $\sim 100 \%$ reaction yield, indicating that the role of oleylamine is to increase the reactivity of the zinc carboxylates.

Figure 1 shows an overview $\mathrm{ZnO}$ NCs prepared with our slow injection protocol. The detailed description of the experimental conditions is presented in the Methods section. Briefly, the zinc oleate/oleylamine precursor is slowly dripped $(0.3 \mathrm{~mL} / \mathrm{min})$ into preheated oleyl alcohol $\left(240-280^{\circ} \mathrm{C}\right)$ under a constant stream of nitrogen flushed through the headspace of the reaction flask. We conducted our reactions using a large excess of oleyl alcohol $(16 \mathrm{~mL}, 50$ mmol) compared to the amount of zinc precursor injected (usually less than $2 \mathrm{mmol}$ in total). The synthesized NCs are generally isotropic, either spheroidal or pyramidal in shape (Figure 1a), with size ranging between $\sim 5$ and $30 \mathrm{~nm}$. The size can be readily controlled through the 
reaction temperature and the amount of precursor used, as it will be thoroughly discussed later in the manuscript. Our $\mathrm{ZnO} \mathrm{NCs}$ are highly crystalline, displaying the commonly observed wurtzite crystal structure (ICDD No. 36-1451, Figure 1b). Moreover, as expected from ZnO, the NCs are highly transparent in the visible range and show a pronounced absorption onset in the near UV region (Figure 1c), associated with the direct band gap of $\mathrm{ZnO}$ at around $3.3 \mathrm{eV}$ (Figure 1d).
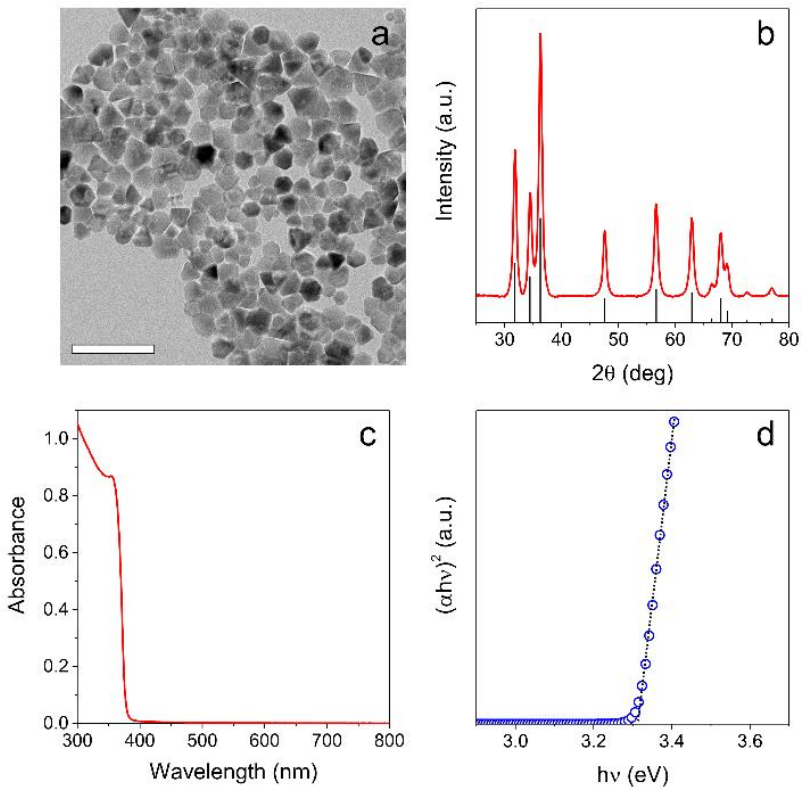

Figure 1. Characterization of typical ZnO NCs synthesized with the slow growth approach. a) TEM image (the scale bar is $100 \mathrm{~nm}$ ). b) XRD pattern (the $\mathrm{ZnO}$ wurtzite reference ICDD No. 36-1451 is highlighted as vertical lines). c) Optical absorption spectra and d) respective Tauc plot for a $\mathrm{ZnO}$ colloidal solution.

After demonstrating the ability to synthesize $\mathrm{ZnO}$ NCs with a slow injection protocol, we explored the parameter space of this colloidal synthesis starting from the investigation of the effect of the reaction temperature and of the amount of precursor added on the properties of $\mathrm{ZnO}$ NCs. Adding slowly the amine/oleate precursor into hot oleyl alcohol triggers the formation of $\mathrm{ZnO} \mathrm{NCs}$ quite reproducibly. However, if we inject just a couple of drops of zinc 
precursor into the alcohol, $\mathrm{ZnO} \mathrm{NCs}$ do not nucleate. We confirmed this through UV-Vis spectroscopy, which shows no presence of the $\mathrm{ZnO}$ absorption edge. By performing a series of slow addition reactions at various temperatures we consistently found that $\mathrm{ZnO} \mathrm{NCs}$ form after injecting $0.2-0.3 \mathrm{mmol}$ of precursor, corresponding to a monomer concentration of at least $0.012 \mathrm{M}$. The formed NCs could then be easily recovered from the mother liquor with common antisolvent precipitation protocols (see Methods). Therefore, a critical amount of precursor is required to trigger the nucleation of the $\mathrm{ZnO} \mathrm{NCs}$. This is consistent with the conventional nucleation and growth theory of nanocrystals (first outlined by LaMer), which demonstrated that a threshold concentration of monomer is required for the solution to become supersaturated and to promote nucleation. ${ }^{40}$ Notably, once this threshold is reached, $\mathrm{ZnO}$ NCs nucleate and stop growing almost instantly. In fact, by monitoring optically the absorption edge of $\mathrm{ZnO}$ we found no change over time (see Figure S1 in the Supporting Information). This suggests that once the critical concentration is reached and the NCs nucleate, the reactivity of our precursor is high, and this enables fast growth of NCs. This is a desirable scenario for our synthesis because it will prevent build-up of unreacted precursor during the slow injection process.

Considering the role played by this critical amount of precursor, we decided to isolate the nucleation phase from the growth by rapidly injecting a small aliquot of precursor $(1 \mathrm{~mL}$, corresponding to $0.33 \mathrm{mmol}$ of zinc giving a monomer concentration of $\sim 0.02 \mathrm{M}$ ) into hot oleyl alcohol and let it react for a few minutes before adding the remainder of the precursor through the slow injection protocol. In this way we could undertake a detailed study of the parameter space of the growth of $\mathrm{ZnO} \mathrm{NCs}$ independent of the nucleation event. All reactions conducted at $240{ }^{\circ} \mathrm{C}$ and above yielded NCs, while reactions carried out below this temperature failed to nucleate $\mathrm{ZnO}$ reproducibly. All materials synthesized above $240{ }^{\circ} \mathrm{C}$ are crystalline and show phase pure wurtzite $\mathrm{ZnO}$ (Figure 2a). The NCs synthesized at higher temperatures are on average bigger than those synthesized at lower temperatures, as commonly observed in 
colloidal syntheses. This is obvious from the TEM images presented in Figure $2 b$-e and from the crystallite size evaluated using the Scherrer formula from the XRD patterns (Figure S2). Unexpectedly, ZnO NCs synthesized at the highest temperature $\left(300{ }^{\circ} \mathrm{C}\right)$ show a slightly smaller crystallite size. TEM analysis of these NCs revealed some evidence of etching (Figure 2e), with many particles showing a concave cone shape, not observed in the other samples. We believe that the excess oleic acid is responsible for the slow dissolution of $\mathrm{ZnO}$ through the formation of zinc oleate at such high temperatures. To prove that, we synthesized a sample in the same exact conditions, but with half the amount of oleic acid. This amount is still enough to form pure oleate precursor, but the "free" oleic acid in the reaction mixture is drastically reduced. We observed a substantial reduction in the degree of etching, and a slightly larger crystallite size, therefore confirming the role played by oleic acid in the slow dissolution of $\mathrm{ZnO}$ (Figure S2 and Figure S3). It is worth noting that similar effects have been observed previously for various metal oxide $\mathrm{NCs}$, including $\mathrm{ZnO}$. ${ }^{41,42,43,44}$
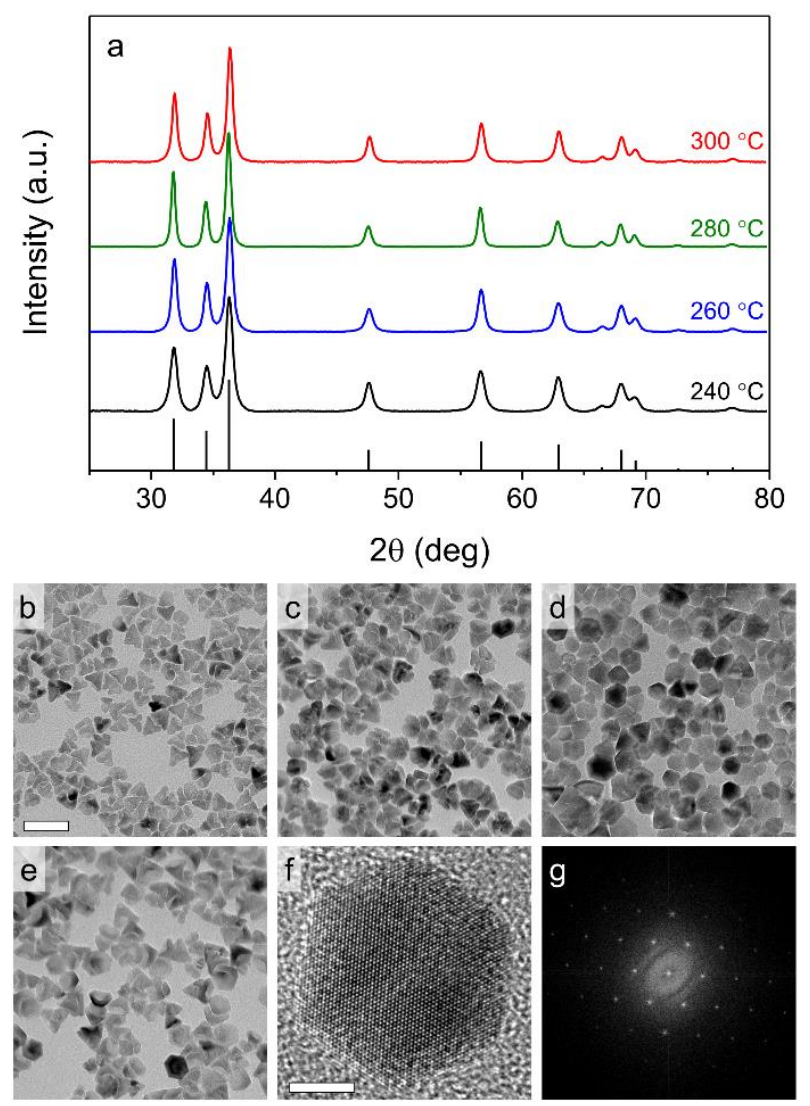
Figure 2. Effect of reaction temperature. a) XRD patterns for $\mathrm{ZnO}$ NCs synthesized at different temperatures. The reference pattern for bulk ZnO (ICDD No. 36-1451) is reported at the bottom. b-e) TEM images of $\mathrm{ZnO}$ NCs synthesized at $240^{\circ} \mathrm{C}$ (b), $260{ }^{\circ} \mathrm{C}$ (c), $280{ }^{\circ} \mathrm{C}$ (d) and $300{ }^{\circ} \mathrm{C}(\mathrm{e})$. The scale bar is $50 \mathrm{~nm}$. f) High resolution TEM of a single $\mathrm{ZnO} \mathrm{NC}$ (scale bar 2 $\mathrm{nm})$ and $(\mathrm{g})$ respective FFT image.

As mentioned earlier, all synthesized particles are crystalline, as confirmed by XRD data as well as by high resolution TEM and the associate Fast Fourier Transform (FFT) presented in Figure $2 \mathrm{f}, \mathrm{g}$. Importantly, while we studied the growth of $\mathrm{ZnO}$ by isolating the nucleation event through the swift injection of the first $0.33 \mathrm{mmol}$ of precursor, we demonstrated that this modification has little or no effect on the final product compared to a conventional continuous addition method, as shown in Figure S2 and Figure S4. Interestingly, the photoluminescence properties of $\mathrm{ZnO} \mathrm{NCs}$ are dependent on the reaction temperature. It is well known that besides the band edge emission, $\mathrm{ZnO} \mathrm{NCs}$ show a distinctive emission band in the visible region associated with a variety of defects, mostly located at the surface, including oxygen vacancies, zinc vacancies and zinc interstitials..$^{45,46,47,48}$ In this study we noted that the defect band is well defined for $\mathrm{ZnO}$ synthesized at high temperatures, while is suppressed in NCs synthesized at lower temperatures (Figure S5). This points to either less defective NCs and/or better defect passivation in NCs synthesized at lower temperatures, which is somewhat unexpected, as higher reaction temperatures are known to reduce the amount of surface defects and the related emission properties in semiconductor NCs, including $\mathrm{ZnO} .{ }^{49,50,51}$

The other parameter other than reaction temperature that is responsible for particle size is the amount of precursor used. It has been reported that for indium oxide the size of the nanocrystals can be accurately controlled through the progressive addition of metal precursors, which preferentially decompose on the surface of pre-formed NCs rather than nucleating 
homogeneously in solution. ${ }^{26,52}$ We performed a series of reactions and analyzed the growth of the NCs at different temperatures by collecting aliquots at different addition times (volumes), and analyzing the optical properties (band gaps) and the size of the NCs by TEM. Representative results are reported in Figure 3. A reduction of band gap is observed by either increasing the reaction temperature or increasing the amount of precursor added, confirming progressive growth of particles (Figure 3a). The band gap values tend to converge to $\sim 3.3 \mathrm{eV}$, the value for bulk $\mathrm{ZnO}$, regardless of the reaction temperature. When a small amount of precursor added (less than $\sim 1 \mathrm{mmol}$ ) the NCs are in the quantum confined regime, while if more precursor is added, the NCs grow beyond quantum confinement and behave optically as bulk $\mathrm{ZnO}$. For reference, all the Tauc plots used to obtain the graph presented in Figure $3 \mathrm{a}$ are in the Supporting Information as Figure S6.

The growth of $\mathrm{ZnO} \mathrm{NCs}$ according to reaction temperature and amount of precursor used is further confirmed by TEM images and the respective size histograms (Figure $3 \mathrm{~b}$ and Figure S7), which show progressive particle growth with the amount of precursor added and on average larger particles at higher reaction temperatures. The size of the NCs can be easily tuned between $\sim 5 \mathrm{~nm}$ to above $25 \mathrm{~nm}$ with our reaction protocol. The size distribution for these $\mathrm{ZnO}$ particles is around $15 \%$ for small particles $(<10 \mathrm{~nm})$ and increases to $\sim 25 \%$ for larger particles (see Table S1 for complete statistics). This broadening of the size distribution could be due to the fact that a small fraction of zinc precursor homogeneously nucleates in solution, rather than contributing to the growth of existing particles. This is a major difference compared to previously reported $\mathrm{In}_{2} \mathrm{O}_{3}$ syntheses where after the nucleation event, the growth happens almost exclusively on existing surfaces, ensuring a narrow particle size distribution $(\sim 10 \%)$ even for large particles. ${ }^{52}$ Moreover, looking at the emission properties, we did not find any substantial difference in photoluminescence of $\mathrm{ZnO} \mathrm{NCs}$ as a function of their size if the synthesis temperature remains the same, except for the expected slight shift of the band edge 
emission towards lower energies (Figure S8). We tried to improve the dispersity in our ZnO NCs by reducing the growth temperature $\left(210-225^{\circ} \mathrm{C}\right)$ after the nucleation event (achieved at $\left.240-260{ }^{\circ} \mathrm{C}\right)$. We noted that even if the size distribution was slightly narrowed, reaching $\sim 15 \%$ at the end of the reaction, both the NC size and the yield of the reaction were lower than expected (Figure S9). This shows that at low growth temperature $\left(<225^{\circ} \mathrm{C}\right)$ the zinc precursor is not reactive enough to contribute substantially to NC growth. We therefore decided not to pursue further these two-temperature reactions and focus on the synthesis at constant temperature, which ensure higher reaction yields even if the distribution of the size of the NCs is slightly broadened.
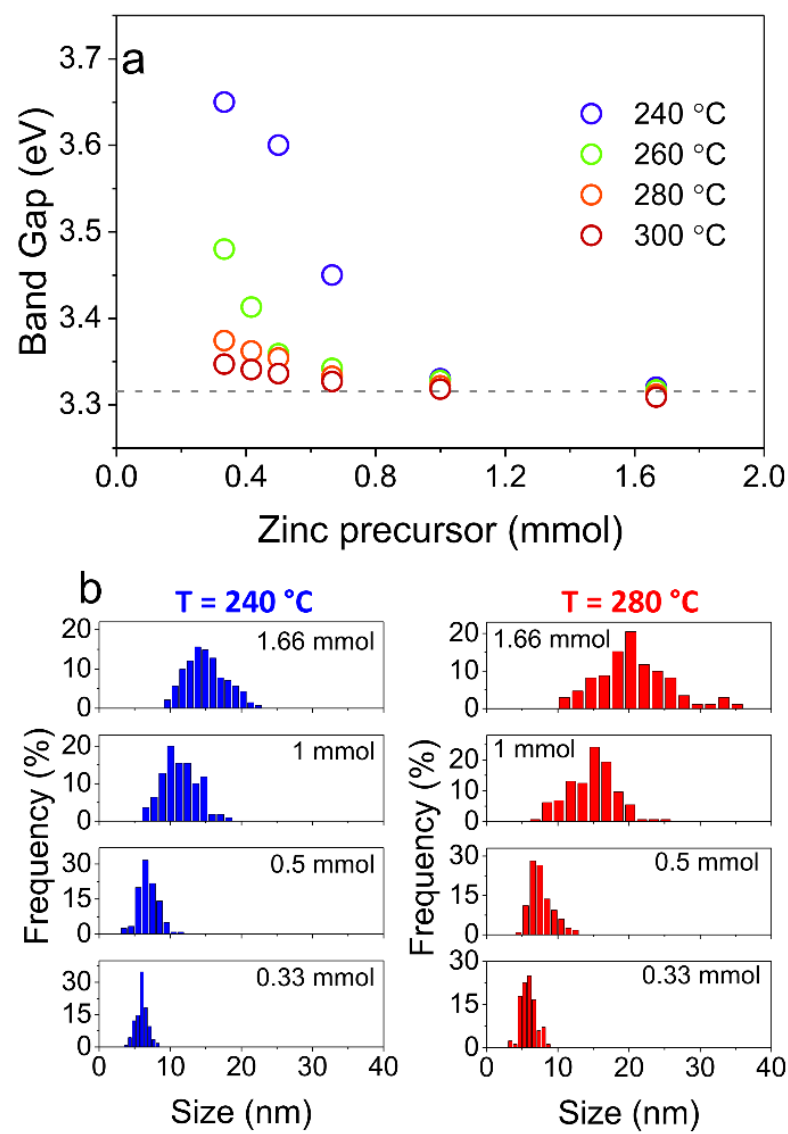

Figure 3. Effect of reaction time and temperature. a) Optical band gap for $\mathrm{ZnO} \mathrm{NCs}$ synthesized at different temperatures and using different amounts of precursor. The dashed horizontal line identifies the bulk band gap of $\mathrm{ZnO}$. b) Size distribution histograms obtained from TEM of NCs synthesized at $240{ }^{\circ} \mathrm{C}$ and $280{ }^{\circ} \mathrm{C}$ with different amounts of zinc precursor. 
As mentioned earlier, in the temperature range we investigated we observed that the nucleation of $\mathrm{ZnO}$ is very fast, and once the precursor is injected into the hot oleyl alcohol it reacts almost immediately. This enables the reaction to be stopped and kept at a set temperature without the further growth of particles. This is shown in Figure S1 for small NCs synthesized with $0.33 \mathrm{mmol}(0.02 \mathrm{M})$ zinc precursor and in Figure S10 where particle synthesized using $1.66 \mathrm{mmol}$ precursor $(0.079 \mathrm{M})$ are kept at the reaction temperature $\left(280{ }^{\circ} \mathrm{C}\right)$ for one hour without adding any more precursor. This stability of ZnO NCs towards prolonged annealing in solution allows for the reliable tracking of the NC sizes (for example with a simple UV-Vis measurement) and if larger particles were required, to re-start the addition. We further confirm this by adding even more precursor, maintaining all the other reaction parameters constant. This is shown in Figure S11 for ZnO NCs synthesized with up to $6.64 \mathrm{mmol}$ precursor (zinc concentration $0.185 \mathrm{M}$ ): the diffraction patterns show sharper peaks with increasing amount of precursor added, indicating larger NC size.

Having described in detail the effect of temperature and amount of precursor on the growth of ZnO NCs using a slow injection method, and showing full tunability in the size of $\mathrm{ZnO}$ NCs, we now focus on the synthesis of doped ZnO. Plasmonic semiconducting NCs, and especially doped metal oxides have recently attracted a lot of interest for a variety of applications in optoelectronics, catalysis and sensing. Most of the literature focuses on ITO due to its sharp LSPR in the near infrared and the ability to synthesize NCs of a specific size, doping level and dopant location within the NCs. ${ }^{27,53,54}$ In this work we focus on doped $\mathrm{ZnO}$, as a cheaper, earth-abundant alternative, which is also still relatively unexplored in the form of colloidal NCs. When $\mathrm{ZnO}$ is doped with aliovalent cations such as $\mathrm{Al}, \mathrm{Ga}$ or $\mathrm{In}$, it can become a degenerate semiconductor showing enhanced electrical conductivity and the rise of a LSPR, 
typically in the mid-IR range. ${ }^{55,56,57,58}$ Here we chose aluminium as the dopant because of its earth abundance, and also because (due to the large difference in ionic radius between divalent zinc and trivalent aluminium) it is the hardest of the trivalent cations mentioned earlier to be incorporated within the $\mathrm{ZnO}$ lattice. ${ }^{59,60}$ We synthesized a series of $\mathrm{Al}$-doped $\mathrm{ZnO}(\mathrm{AZO}) \mathrm{NCs}$ with different dopant amounts by substituting a fraction of zinc precursor with Al precursor, and performing the reaction in the same way as for the undoped $\mathrm{ZnO}$ (see Methods for details).

A distinctive change in color of the synthesized NCs from colorless to green-blue is observed when $\mathrm{Al}$ is present, with the color being more intense at higher doping levels. This color has been observed previously in doped $\mathrm{ZnO},{ }^{60,61}$ and is common in other plasmonic metal oxides such as ITO, $\mathrm{WO}_{3-\mathrm{X}}$, and Sb-doped $\mathrm{SnO}_{2} \cdot{ }^{62,63,64}$ Figure 4a shows the optical absorption spectra in the visible and near infrared for AZO NCs synthesized with different Al doping level (5, 10 and 20 atomic \% nominal). A strong absorption in the infrared is observed, corresponding to the rise of the LSPR peak. In addition, a progressive widening of the optical band gap is observed, which is ascribed to the Burstein-Moss effect, given that the NCs are well outside the quantum confinement regime. ${ }^{49}$ The nature of the NIR absorption has been confirmed with FTIR (Figure S12) which shows the LSPR peak centered between $\sim 5$ and $8 \mu \mathrm{m}$ depending on the doping level. From the position of the plasmon we may roughly estimate the free carrier concentration using simple Mie-Drude formalism, assuming spherical particles and homogeneous doping. A detailed description of the equation used is reported elsewhere. ${ }^{60}$ Despite the assumptions made, this model gives a metric to estimate the carrier concentrations and therefore the amount of active dopants within $\mathrm{ZnO}$. In addition, through compositional analysis we evaluated the amount of Al incorporated into the NCs. Figure $4 \mathrm{~b}$ shows a plot of Al doping amount $v s$. carrier concentration. The carrier concentration is as high as $10^{20} \mathrm{~cm}^{-3}$ for the NCs synthesized with the highest doping, which is consistent with previous results on doped $\mathrm{ZnO} .{ }^{65,60}$ Moreover, the doping efficiency (dopant effectively incorporated within $\mathrm{ZnO}$ 
with respect to the amount of precursor used) is $\sim 40 \%$, regardless of the actual doping level (see also Table S2). This is a remarkable result, considering the reported low reactivity of A1 precursors combined with the stresses induced in the $\mathrm{ZnO}$ lattice by the small $\mathrm{Al}^{3+}$ ions. Therefore, with this synthetic method we were able to achieve tunable doping levels up to $10 \%$, without the segregation of any impurity phases, as confirmed by XRD analyses (Figure S13). However, we did notice a progressive broadening of the LSPR peak with increasing dopant concentration. The measured full width at half maximum (FWHM) values are $\sim 1200 \mathrm{~cm}^{-1}$, $\sim 1450 \mathrm{~cm}^{-1}$ and $\sim 1900 \mathrm{~cm}^{-1}$ for AZO5, AZO10 and AZO20, respectively. This broadening can be indicative of increased ionized impurity scattering, which is common in plasmonic metal oxides, especially at high doping levels. ${ }^{14}$ In addition, doping variations between individual NCs, and shape effects in faceted NCs can also be held accountable for such broadened LSPR when measured for an ensemble of particles. ${ }^{66}$

We analyzed the synthesized AZO NCs using TEM (see Figure S14) and we noted that the presence of small amounts of Al does not affect significantly the NC size or shape. This is in contrast with what was observed previously. ${ }^{65,60}$ However, for large Al doping levels, the NCs become highly polydisperse and irregular, possibly due to the stresses caused by excess Al to the $\mathrm{ZnO}$ crystals, which heavily affect their growth. 

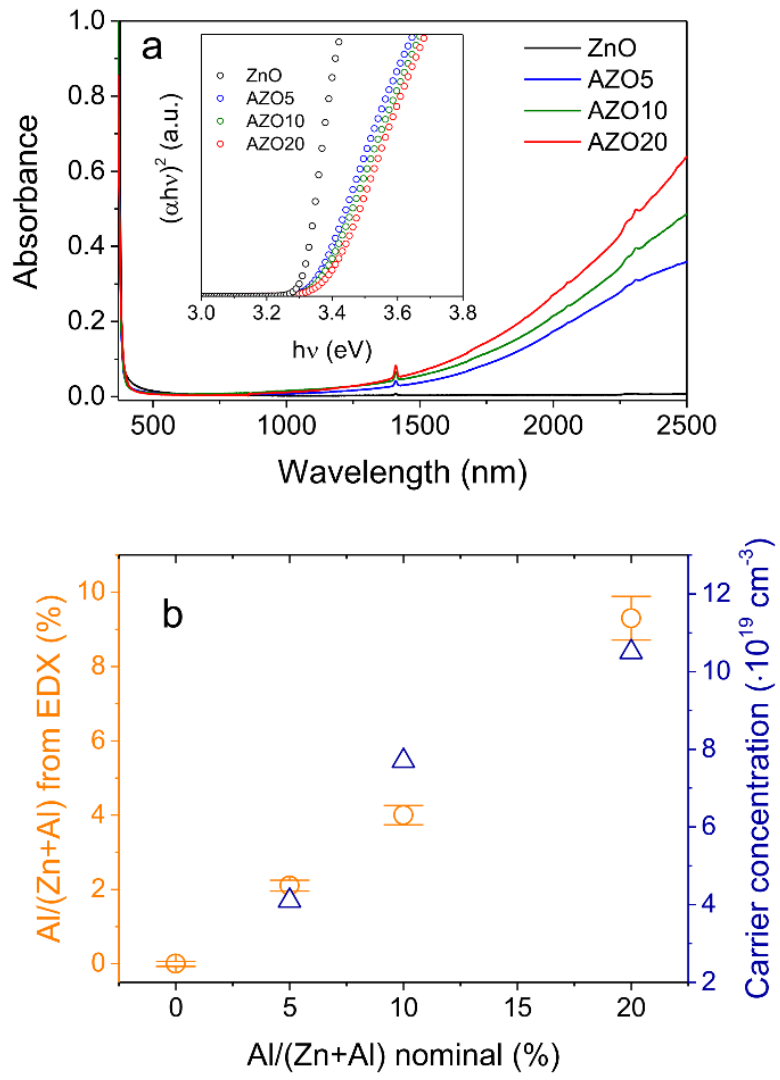

Figure 4. Synthesis of Al-doped ZnO NCs. a) UV-Vis-NIR spectra of ZnO NCs doped with different amounts of Al. The inset shows the respective Tauc plots. b) Charge carrier concentration evaluated from the LSPR frequency and nominal vs. real (from EDX) Al concentration for $\mathrm{ZnO} \mathrm{NC}$ doped with different amounts of aluminum.

After establishing the reaction protocol for highly doped $\mathrm{ZnO} \mathrm{NCs}$, we decided to investigate the kinetics of doping using this continuous growth method. As discussed earlier, undoped NCs do not change over time when the addition of the precursor is stopped, i.e. the precursor reacts almost instantly and completely. However, in the case of doped NCs, the kinetics are slightly different. Figure 5 shows the results of a kinetic study conducted on an Aldoped $\mathrm{ZnO}$ sample (AZO10, 4\% actual doping), in which aliquots are collected over time, even up to 4 hours after the addition of the precursor has stopped, while the NCs are kept at the reaction temperature $\left(280^{\circ} \mathrm{C}\right)$. A progressive increase in the IR absorption (plasmon) is seen 
up to 2 hours after stopping the addition of the precursor (Figure 5a). This is likely due to both the Al precursor being less reactive than the zinc precursor and the thermodynamic barrier to dopant incorporation within the host lattice. A similar behavior has been observed previously. ${ }^{65}$ This is exemplified in Figure 5b, which shows the amount of precursor added over time (blue line, left vertical axis) and the recorded absorbance value in the near IR for the corresponding AZO NCs (open circles with colors matching those of the spectra in Figure 5a, right vertical axis). We can clearly see how the IR absorption rapidly increases during the injection stage (see also Figure S15 for a zoomed view of the early stages of growth), and keeps increasing during the annealing stage, albeit at a slower rate, until eventually it plateaus. Such a behavior can be explained with the low reactivity and corresponding slow incorporation rate of aluminum. In fact, a very weak IR absorption is observed for small $\mathrm{ZnO} \mathrm{NCs}$ at the beginning of the reaction. However, the IR absorption increases substantially during the course of the injection, and after the injection is concluded. This may point towards a higher dopant concentration at the surface of the NCs, which is also another factor that could be responsible for the increased broadening of the LSPR at high doping levels. Interestingly, looking at the TEM of NCs at different stages of the reaction (Figure 5d-g) we can see how doped particles show a slight "delay" in growth, with smaller particles being present even at the latest stages of the reaction. This is clear from the size distribution histograms which does not show much change up to $0.66 \mathrm{mmol}(0.037 \mathrm{M})$, and also shows a largely polydisperse, almost bimodal distribution at the end of the slow addition of the precursor. Interestingly, this size distribution sharpens when annealing the NCs in the mother liquor at the reaction temperature for longer times. This suggests a slower growth rate for doped $\mathrm{NCs}$ compared to undoped $\mathrm{ZnO}$, possibly due to the $\mathrm{Al}$-induced stresses in the host wurtzite lattice. Therefore, this prolonged heating after finishing the addition of the precursor has the dual effect of promoting additional dopant incorporation and of properly growing the NCs to their final size. 

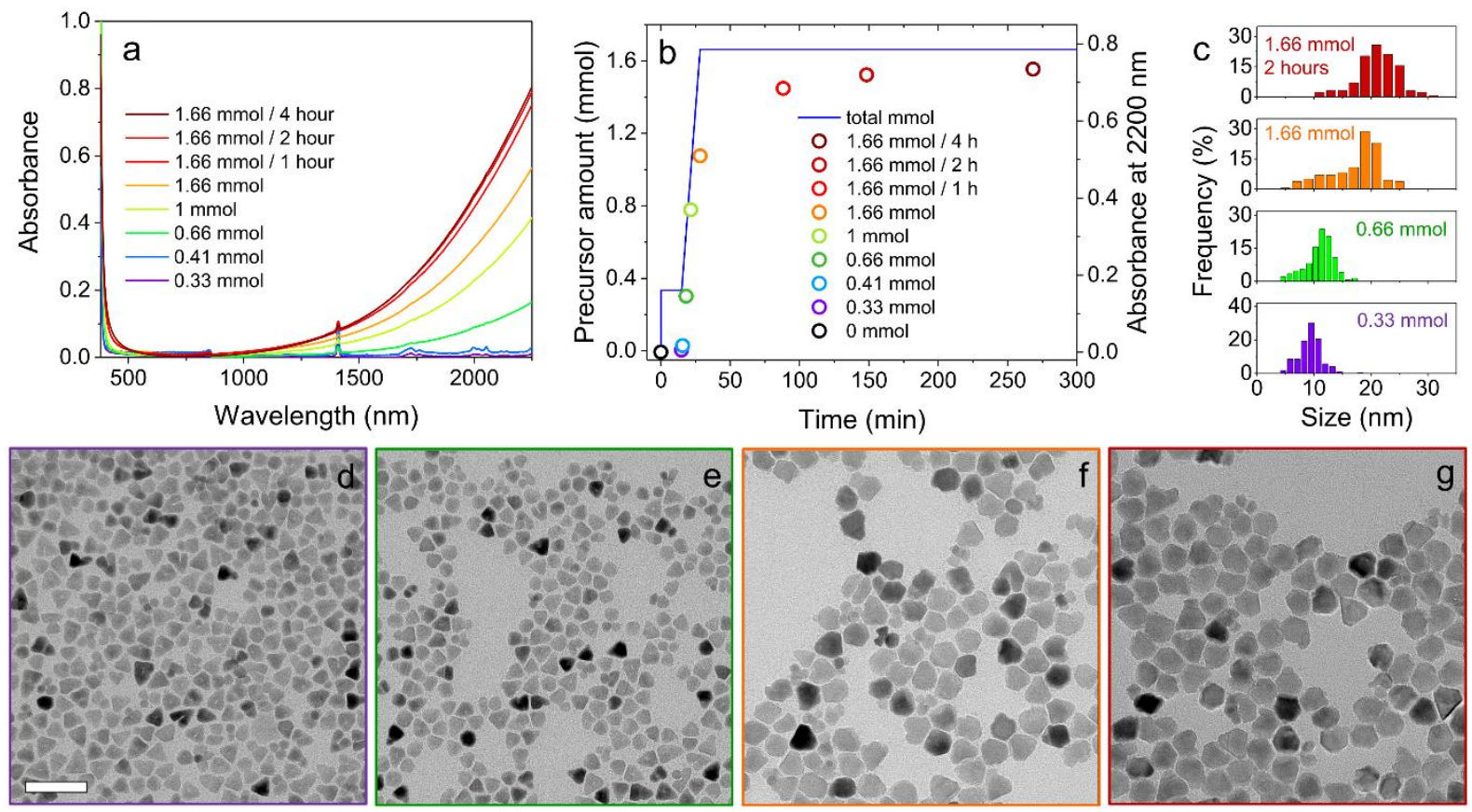

Figure 5. Growth kinetics of AZO NCs. a) UV-vis-NIR spectra of AZO10 NCs synthesized with different precursor amounts and different reaction times. b) Absorbance value monitored at $2200 \mathrm{~nm}$ as a function of the reaction time and the amount of precursor added. c) Size distribution histograms for AZO10 NCs at different stages of the reaction. d-g) TEM images for AZO10 NCs synthesized with $0.33 \mathrm{mmol}(0.02 \mathrm{M}$, d), $0.66 \mathrm{mmol}(0.037 \mathrm{M}, \mathrm{e}), 1.66 \mathrm{mmol}$ $(0.079 \mathrm{M}, \mathrm{f}, \mathrm{g})$ of precursor. Sample $(\mathrm{g})$ has been let react for 2 hours from the end of the injection. Scale bar is $50 \mathrm{~nm}$ and is common to all TEM images.

To prove the versatility of our reaction system, we synthesized ZnO NCs doped with other trivalent cations, namely Ga and In. In all cases we were successful in synthesizing phase pure, plasmonic $\mathrm{ZnO}$ NCs. XRD results show pure phase wurtzite $\mathrm{ZnO}$ for both Ga-doped and In-doped $\mathrm{ZnO}$ (Figure S16), similar to what we observed for both Al-doped $\mathrm{ZnO}$ and undoped $\mathrm{ZnO}$. Interestingly, the (002) diffraction peak of Ga-doped $\mathrm{ZnO}$ is much sharper, indicating a larger crystallite size along the (002) direction, corresponding to the $c$-axis of the hexagonal wurtzite lattice. This indicates the presence of elongated, rod-like particles, which was confirmed with TEM analyses (Figure S17). This is not the case for IZO NCs, which are 
spheroidal and isotropic. Elemental analysis confirms the presence of trivalent dopants within $\mathrm{ZnO}$, and shows that the doping efficiency for $\mathrm{Ga}$ and In is larger than that for $\mathrm{Al}$, as expected from their ionic radius. ${ }^{59}$ Specifically, for a nominal 5\% doping, Al-doped ZnO NCs show a $2 \%$ doping level (efficiency of $40 \%$ ), while Ga- $\mathrm{ZnO}$ have a $2.8 \%$ doping level (efficiency of $56 \%$ ) and $\mathrm{In}-\mathrm{ZnO}$ has a 3.9\% doping level (efficiency of 78\%). These details are summarized in Table S2 in the supporting information. Analyzing the optical properties of these doped NCs in colloidal solutions (Figure S18), we can see a clear plasmonic absorption for all doped NCs, with In-doped $\mathrm{ZnO}$ having the most intense absorption, as expected. It is worth noting that Ga$\mathrm{ZnO}$ NCs have a lower absorption in the near IR compared to $\mathrm{Al}-\mathrm{ZnO}$, despite having a larger dopant fraction. This is not surprising considering the shape of the NCs: while AZO NCs are reasonably isotropic, GZO NCs are elongated, with a large aspect ratio. This will cause a prominent red shift of the longitudinal plasmon, as predicted by Mie-Gans theory, resulting in lower absorption intensity in the near infrared. ${ }^{67}$

To demonstrate the full potential of this reaction system, we performed a gram-scale synthesis, as proof that our optimized method is readily scalable. We scaled up both the amount of precursor $(15 \mathrm{mmol})$ and the amount of oleyl alcohol $(160 \mathrm{~mL})$ achieve a final metal concentration of $0.073 \mathrm{M}$. We also adjusted the injection rate in order to keep it consistent with the standard "small scale" syntheses (see Methods for details), and we used Al-doped ZnO as a model system. As shown in Figure 6, after continuously dripping the $15 \mathrm{mmol}(45 \mathrm{~mL})$ of metal precursor into oleyl alcohol at $280{ }^{\circ} \mathrm{C}$ we achieved deep-blue colored powders, which are highly crystalline and possess an intense infrared absorption. Compared to a small batch reaction, the NCs are slightly more polydisperse, however the particle size is identical, confirming the robustness of our reaction for gram-scale syntheses. Importantly, we analyzed the reaction yield by weighing the dried product (Figure 6a), and quantifying the amount of organic material using TGA (Figure S19): our reaction produced $\sim 1.37 \mathrm{~g}$ of dry powders, which 
correspond to $\sim 1.21 \mathrm{~g}$ of purely inorganic material, considering a weight loss of $\sim 11.5 \%$ associated with the decomposition of the organic surface ligands. Since the reaction was conducted using $15 \mathrm{mmol}$ of metal precursor, we would have expected $\sim 1.22 \mathrm{~g}$ of inorganic product. Therefore, we can conclude that the reaction yield is remarkably close to $100 \%$.
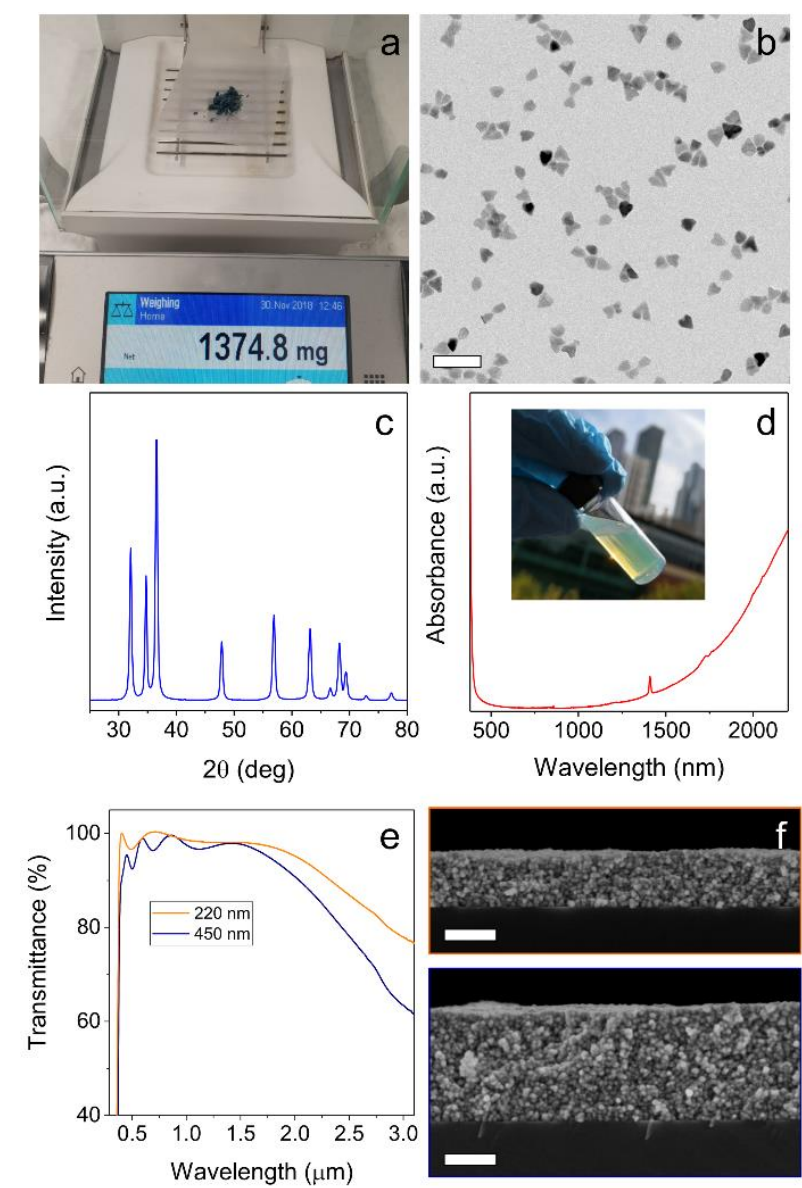

Figure 6. Large scale reaction of AZO10 NCs. a) Picture of the dried product recovered from the large scale reaction. b) TEM image (scale bar $100 \mathrm{~nm}$ ) of the NCs. c) XRD pattern. d) UVVis-NIR spectrum. e) Transmittance spectra and f) SEM cross-sectional images of two AZO10 nanocrystalline films of different thickness (scale bar $200 \mathrm{~nm}$ ).

The ability to assemble colloidal NCs into high quality thin films is one of the main features sought after for the use of NCs within various devices, including optoelectronics. Owing to their hydrophobic surface ligands, these $\mathrm{ZnO}$ and doped $\mathrm{ZnO} \mathrm{NCs}$ can be readily 
dispersed in several organic solvents such as toluene, hexane, and octane, at high concentration (>100 mg/mL). These NC "inks" can then be used for thin film deposition with conventional solution-based methods. Figures $6 \mathrm{e}$ and $6 \mathrm{f}$ show the optical transmission spectra and the crosssectional SEM of two AZO10 films (nominal Al concentration 4\%) deposited by layer-by-layer spin coating and annealing cycles, to achieve a thickness of $\sim 220 \mathrm{~nm}$ (2 layers) and $450 \mathrm{~nm}$ (4 layers). The AZO coatings are highly transparent in the visible range, and show a prominent NIR absorption, confirming that the colloidal NCs properties are readily transferred to the thinfilm NC assemblies. After correction for the substrate (glass) absorption, the AZO films show exceptional transparency in the visible range, achieving $98.5 \%$ and $97 \%$ average transmittance in the 400-1200 nm range for the 220 and $450 \mathrm{~nm}$ thick film, respectively. The quality of the thin film is indicated by the clear interference fringes and is confirmed by cross sectional SEM images (Figure 6f), which show uniform and homogeneous assembly of AZO NCs (see also Figure S20 for additional SEM images). Preliminary electrical measurements demonstrate that these NC assemblies are indeed conductive, showing resistivity values of the order of $10^{3} \Omega \cdot \mathrm{cm}$ after annealing in air, and a drop of three orders of magnitude down to few $\Omega \cdot \mathrm{cm}$ after annealing in forming gas. These results, even if obtained for non-optimized conditions, are on par with the current literature values for NC-based, Al-doped $\mathrm{ZnO}$ films, highlighting the promise of our AZO NCs. The ability to use these colloidal NCs to fabricate coatings of optical quality with enhanced conductivity makes our reaction method very appealing for the scalable production of $\mathrm{ZnO}$-based materials for use in devices including (photo)catalysts, solar cells, LEDs, electrochromics and sensors.

\section{CONCLUSION}


In conclusion, we have presented a continuous growth reaction for zinc oxide colloidal nanocrystals which enables the production of $\mathrm{ZnO}$ with highly controllable size and doping. The slow addition of a solution of zinc oleate, excess oleic acid and additional oleylamine into hot oleyl alcohol triggers the formation of zinc oxide through an esterification reaction between the zinc precursor and the alcohol. The method developed here entails the slow but continuous addition of precursor, favoring a heterogeneous layer-by-layer growth rather than homogeneous nucleation in solution. Through this strategy, simply controlling the reaction temperature and the amount of precursor used permits access to $\mathrm{ZnO}$ nanocrystals with size tunable from $\sim 5 \mathrm{~nm}$ to $\sim 25 \mathrm{~nm}$ and above. Moreover, doped nanocrystals could be readily synthesized by substituting some of the zinc precursor with appropriate dopant oleates. This was demonstrated through the synthesis of plasmonic $\mathrm{ZnO}$ nanocrystals doped with either aluminium, gallium or indium. In addition, gram-scale syntheses with $100 \%$ reaction yield are presented, validating the scalability of our reaction protocols. Finally, thin films with outstanding quality (visible transmittance $>95 \%$ ) and enhanced electrical conductivity could be easily fabricated from our Al-doped $\mathrm{ZnO}$ nanocrystal inks. These results constitute a marked advancement in the synthesis of pure and doped $\mathrm{ZnO}$ nanocrystals with controllable properties, and will help translating laboratory-scale achievements into commercial technologies.

\section{METHODS}

\section{Materials}

Zinc acetate dihydrate (99\%), oleic acid (OA, 90\%.), oleylamine (OLA, 70\%), and tetrachloroethylene (TCE, 99.5\%) were supplied by Sigma-Aldrich. Aluminum acetylacetonate $\left(\mathrm{Al}(\mathrm{Acac})_{3}, 99 \%\right)$ gallium acetylacetonate $\left(\mathrm{Ga}(\mathrm{Acac})_{3}, 99.99 \%\right)$ indium acetylacetonate $\left(\operatorname{In}(\mathrm{Acac})_{3}, 99.99 \%\right)$ were supplied by Strem Chemicals. Oleyl alcohol (8085\%) was supplied by Alfa Aesar. Toluene (99.5\%), ethanol (99.5\%), methanol (99.8\%) and 
acetone $(99.5 \%)$ were purchased from Univar. All chemicals were used without further purification.

\section{Nanocrystal synthesis}

In a typical reaction, $16 \mathrm{~mL}$ oleyl alcohol is loaded in a $100 \mathrm{~mL}$ three-neck round bottom flask (RBF) and connected to a heating mantle. One of the side necks of the RBF is sealed with a rubber septum and a thermocouple and a 19-gauge needle are pierced through it. The needle is connected to a nitrogen line and the RBF is constantly purged with 8-10 L/h of nitrogen flow. The central neck is sealed with a rubber septum and used for precursor injection (see below). The other side neck is connected to an elbow joint and a short piece of tubing that act as a drain to remove excess water formed during the reaction. This setup avoids the build-up of excess condensation around the necks of the RBF. The oleyl alcohol is then heated to the desired reaction temperature under a constant flow of nitrogen. In the meantime, a $50 \mathrm{~mL} \mathrm{RBF}$ is loaded with $2.4 \mathrm{mmol}$ of the metal precursor and $4.8 \mathrm{~mL}$ of oleic acid. In the case of doped NCs, the precursor is composed of a mixture of zinc acetate and dopant acetylacetonate, and the dopant (D) nominal concentration is defined as

$$
D(\%)=\frac{n_{D}}{\left(n_{Z n}+n_{D}\right)} \times 100
$$

where $n$ is the number of moles. Doped NCs are labelled AZO $X$, GZOX and IZOX (for Al-, Ga- and In- doped $\mathrm{ZnO}$, respectively), where $X$ is the dopant nominal concentration. The RBF is connected to a Schlenk line and heated under vacuum $\left(3-5 \cdot 10^{-3}\right.$ mbar) to $110^{\circ} \mathrm{C}$ and kept at temperature for 20 minutes. The RBF is then backfilled with nitrogen, and OLA $(2.4 \mathrm{~mL})$ is added. The precursor is further degassed for 5 minutes at $100{ }^{\circ} \mathrm{C}$, and then kept at temperature under a nitrogen blanket for further use. Once the oleyl alcohol reached the desired temperature, the precursor solution was loaded into a glass syringe equipped with a long 16- 
gauge needle and slowly injected into the hot alcohol through the center neck using a syringe pump at a rate of $0.3 \mathrm{~mL} / \mathrm{min}$. A photo of the reaction setup is shown in Figure S21. For consistency across different reactions, the slow injection was stopped after a total of $5 \mathrm{~mL}$ (1.66 mmol) precursor was added. One hour after the addition of the precursor is finished, the heater is turned off and the NC suspension is let cool to room temperature naturally under the nitrogen flow. Excess ethanol is then added to precipitate the NCs, which are then centrifuged (4400 rpm, 5 minutes) and resuspended in toluene or TCE. This process is repeated 2-3 times to further purify the NCs. During the reactions, aliquots are taken at different stages and precipitated with excess ethanol. At the very early stages of the NC growth, methanol had also to be used to cause flocculation of small NCs. As discussed in the manuscript, to isolate growth from nucleation a series of reactions was performed with the rapid injection of $1 \mathrm{~mL}(0.33$ mmol) of the metal precursor the hot oleyl alcohol under strong stirring and let react for 15 minutes. After this step, the slow injection protocol was carried out as described earlier. Deviations from the typical reaction conditions including the use of less oleic acid $(2.4 \mathrm{mmol}$ instead of $4.8 \mathrm{mmol}$, Figure S3), the use of more precursor in the slow injection process (up to 6.6 mmol, Figure S11) and the use of two growth temperatures in a single reaction (Figure S9).

To synthesize AZO10 NCs in large scale, the synthesis was adapted as follows. The metal precursor was prepared by dissolving zinc acetate $(14.4 \mathrm{mmol})$ and $\mathrm{Al}(\mathrm{Acac})_{3}(1.6 \mathrm{mmol})$ in $32 \mathrm{~mL}$ oleic acid, degassed at $110{ }^{\circ} \mathrm{C}$ for one hour, followed by addition of $16 \mathrm{~mL}$ OLA and further degassed at temperature for 15 minutes. Excess oleyl alcohol $(160 \mathrm{~mL})$ was heated in a $250 \mathrm{~mL}$ RBF under strong stirring and after the desired temperature was reached, $45 \mathrm{~mL}(15$ mmol) precursor was slowly added using a syringe pump. In order to maintain the same NC growth rate, the injection rate was increased to $3 \mathrm{~mL} / \mathrm{min}$. Moreover, the nitrogen flowrate was increased to $\sim 20 \mathrm{~L} / \mathrm{h}$, to facilitate the removal of the excess water vapor formed. A parallel reaction conducted with the swift injection of $5 \mathrm{~mL}(1.66 \mathrm{mmol})$ metal precursor to isolate the 
nucleation event, followed by the slow addition of $40 \mathrm{~mL}(13.33 \mathrm{mmol})$ precursor resulted in almost identical NCs.

AZO thin films were deposited from concentrated $(\sim 50 \mathrm{mg} / \mathrm{mL})$ AZO NC suspensions in toluene using spin coating with a spinning speed of $3000 \mathrm{rpm}$ for 5 seconds, followed by 5000 rpm for 30 seconds. The deposited films were stabilized at $150{ }^{\circ} \mathrm{C}$ in air on a hot plate for 5 minutes, and additional layers were deposited with the same procedure if required. Post annealing was conducted at $300{ }^{\circ} \mathrm{C}$ in air for 1 hour. Additional annealing in forming gas at $500{ }^{\circ} \mathrm{C}$ for 1 hour was conducted to increase the conductivity of the AZO coatings.

\section{Characterization techniques}

Transmission electron microscopy (TEM) images of NCs deposited on carbon-coated copper grids were acquired on a JEOL $2100 \mathrm{~F}$ microscope operated at $200 \mathrm{kV}$. The images were analyzed using the ImageJ software. X-ray diffraction (XRD) patterns of dried NCs powders were collected using a Bruker D4 Endeavor diffractometer equipped with a $\mathrm{Cu}-\mathrm{K} \alpha$ radiation source and operated at $40 \mathrm{kV}$ and $35 \mathrm{~mA}$. The crystallite size was evaluated with the Scherrer relationship using the full width at half-maximum of the diffraction peaks fitted using PseudoVoigt functions. For each sample the extracted size values from each of the 11 diffraction peaks detected between 20 and 80 degrees were averaged and the error was calculated as one standard deviation. Optical absorption spectra of NCs dispersed in tetrachloroethylene (TCE) were obtained on Agilent Cary60 UV-Vis and Cary7000 UV-Vis-NIR spectrophotometers. Photoluminescence (PL) spectra were acquired with a Horiba Jobin Yvon Fluoromax-4 fluorometer with an excitation wavelength of $330 \mathrm{~nm}$. Fourier transform infrared (FTIR) spectroscopy of NCs was carried out using a Perkin-Elmer Frontier spectrometer equipped with a Pike GladiATR attenuated total reflectance (ATR) stage. A drop of NCs dispersed in TCE was deposited on the ATR crystal and the measurement was taken upon TCE evaporation. 
Thermogravimetric analysis (TGA) was conducted on a Perkin Elmer Pyris 1 instrument at a heating rate of $5{ }^{\circ} \mathrm{C} / \mathrm{min}$ in air. Energy dispersive X-ray (EDX) spectra were acquired on a Nova 200 NanoSEM with a voltage of $15 \mathrm{kV}$. Scanning electron microscopy (SEM) images were acquired on a FEI Verios 460L SEM operated at $2 \mathrm{kV}$ and $25 \mathrm{pA}$.

\section{ASSOCIATED CONTENT}

Supporting Information. Additional characterizations and data (Figure S1-S21 and Table S1S2): UV-Vis-NIR, FTIR and PL spectra; XRD patterns; TEM and SEM images; size distribution histograms; crystallite size values; TGA scan; photo of the reaction setup; and additional tables. This material is available free of charge via the Internet at http://pubs.acs.org

\section{AUTHOR INFORMATION}

\section{Corresponding Author}

*enrico.dellagaspera@rmit.edu.au (EDG)

\section{Author Contributions}

The manuscript was written through contributions of all authors. All authors have given approval to the final version of the manuscript.

\section{ACKNOWLEDGMENT}

EDG and SJB thank RMIT University for a Vice Chancellor Fellowship. The Australian Research Council is acknowledged for supporting this work through the following grants DE170100164 (EDG), DP190101864 (EDG, AT) and DE150100427 (JvE). The authors acknowledge the facilities and the technical assistance of the RMIT University's Microscopy and Microanalysis Facility (RMMF). 


\section{REFERENCES}

1. Kovalenko, M. V.; Manna, L.; Cabot, A.; Hens, Z.; Talapin, D. V.; Kagan, C. R.; Klimov, V. I.; Rogach, A. L.; Reiss, P.; Milliron, D. J.; Guyot-Sionnnest, P.; Konstantatos, G.; Parak, W. J.; Hyeon, T.; Korgel, B. A.; Murray, C. B.; Heiss, W., Prospects of Nanoscience with Nanocrystals. ACS Nano 2015, 9 (2), 1012-1057.

2. Goesmann, H.; Feldmann, C., Nanoparticulate Functional Materials. Angewandte Chemie International Edition 2010, 49 (8), 1362-1395.

3. Choi, J.-H.; Wang, H.; Oh, S. J.; Paik, T.; Sung, P.; Sung, J.; Ye, X.; Zhao, T.; Diroll, B. T.; Murray, C. B.; Kagan, C. R., Exploiting the colloidal nanocrystal library to construct electronic devices. Science 2016, 352 (6282), 205-208.

4. Brus, L. E., Electron-electron and electron-hole interactions in small semiconductor crystallites: The size dependence of the lowest excited electronic state. The Journal of Chemical Physics 1984, 80 (9), 4403-4409.

5. Murray, C. B.; Norris, D. J.; Bawendi, M. G., Synthesis and characterization of nearly monodisperse $\mathrm{CdE}$ ( $\mathrm{E}=$ sulfur, selenium, tellurium) semiconductor nanocrystallites. Journal of the American Chemical Society 1993, 115 (19), 8706-8715.

6. Thanh, N. T. K.; Maclean, N.; Mahiddine, S., Mechanisms of Nucleation and Growth of Nanoparticles in Solution. Chemical Reviews 2014, 114 (15), 7610-7630.

7. Gu, K. S.; Taeghwan, H., Formation Mechanisms of Uniform Nanocrystals via Hot-Injection and Heat-Up Methods. Small 2011, 7 (19), 2685-2702.

8. van Embden, J.; Chesman, A. S. R.; Jasieniak, J. J., The Heat-Up Synthesis of Colloidal Nanocrystals. Chemistry of Materials 2015, 27 (7), 2246-2285.

9. Owen, J.; Brus, L., Chemical Synthesis and Luminescence Applications of Colloidal Semiconductor Quantum Dots. Journal of the American Chemical Society 2017, 139 (32), 1093910943.

10. Talapin, D. V.; Lee, J.-S.; Kovalenko, M. V.; Shevchenko, E. V., Prospects of Colloidal Nanocrystals for Electronic and Optoelectronic Applications. Chemical Reviews 2010, 110 (1), 389458.

11. Ochsenbein, S. T.; Gamelin, D. R., Quantum oscillations in magnetically doped colloidal nanocrystals. Nature Nanotechnology 2010, 6, 112.

12. Pradhan, N.; Das Adhikari, S.; Nag, A.; Sarma, D. D., Luminescence, Plasmonic, and Magnetic Properties of Doped Semiconductor Nanocrystals. Angewandte Chemie International Edition 2017, 56 (25), 7038-7054.

13. Della Gaspera, E.; Guglielmi, M.; Perotto, G.; Agnoli, S.; Granozzi, G.; Post, M. L.; Martucci, A., CO optical sensing properties of nanocrystalline $\mathrm{ZnO}-$ Au films: Effect of doping with transition metal ions. Sensors and Actuators B: Chemical 2012, 161 (1), 675-683.

14. Lounis, S. D.; Runnerstrom, E. L.; Llordés, A.; Milliron, D. J., Defect Chemistry and Plasmon Physics of Colloidal Metal Oxide Nanocrystals. The Journal of Physical Chemistry Letters 2014, 5 (9), 1564-1574.

15. Della Gaspera, E.; Griggs, J.; Ahmed, T.; Walia, S.; Mayes, E. L. H.; Calzolari, A.; Catellani, A.; van Embden, J., Augmented band gap tunability in indium-doped zinc sulfide nanocrystals. Nanoscale 2019, 11 (7), 3154-3163.

16. Song, J.; Kulinich, S. A.; Li, J.; Liu, Y.; Zeng, H., A General One-Pot Strategy for the Synthesis of High-Performance Transparent-Conducting-Oxide Nanocrystal Inks for All-Solution-Processed Devices. Angewandte Chemie International Edition 2015, 54 (2), 462-466.

17. Kim, B. H.; Staller, C. M.; Cho, S. H.; Heo, S.; Garrison, C. E.; Kim, J.; Milliron, D. J., High Mobility in Nanocrystal-Based Transparent Conducting Oxide Thin Films. ACS Nano 2018, 12 (4), 3200-3208. 
18. Sturaro, M.; Della Gaspera, E.; Michieli, N.; Cantalini, C.; Emamjomeh, S. M.; Guglielmi, M.; Martucci, A., Degenerately Doped Metal Oxide Nanocrystals as Plasmonic and Chemoresistive Gas Sensors. ACS Applied Materials \& Interfaces 2016, 8 (44), 30440-30448.

19. Sakamoto, M.; Kawawaki, T.; Kimura, M.; Vequizo, J. J. M.; Matsunaga, H.; Ranasinghe, C. S. K.; Yamakata, A.; Matsuzaki, H.; Furube, A.; Teranishi, T., Clear and transparent nanocrystals for infrared-responsive carrier transfer. Nature Communications 2019, 10 (1), 406.

20. Zhang, L.; Xia, Y., Scaling up the Production of Colloidal Nanocrystals: Should We Increase or Decrease the Reaction Volume? Advanced Materials 2014, 26 (16), 2600-2606.

21. Niederberger, M. P., Nicola, Metal Oxide Nanoparticles in Organic Solvents: Synthesis, Formation, Assembly and Application. Springer: Berlin and Heidelberg, Germany, 2009.

22. Chen, Y.; Johnson, E.; Peng, X., Formation of Monodisperse and Shape-Controlled MnO Nanocrystals in Non-Injection Synthesis: Self-Focusing via Ripening. Journal of the American Chemical Society 2007, 129 (35), 10937-10947.

23. Chen, Y.; Kim, M.; Lian, G.; Johnson, M. B.; Peng, X., Side Reactions in Controlling the Quality, Yield, and Stability of High Quality Colloidal Nanocrystals. Journal of the American Chemical Society 2005, 127 (38), 13331-13337.

24. Cozzoli, P. D.; Kornowski, A.; Weller, H., Low-Temperature Synthesis of Soluble and Processable Organic-Capped Anatase TiO2 Nanorods. Journal of the American Chemical Society 2003, 125 (47), 14539-14548.

25. Ito, D.; Yokoyama, S.; Zaikova, T.; Masuko, K.; Hutchison, J. E., Synthesis of LigandStabilized Metal Oxide Nanocrystals and Epitaxial Core/Shell Nanocrystals via a Lower-Temperature Esterification Process. ACS Nano 2014, 8 (1), 64-75.

26. Jansons, A. W.; Plummer, L. K.; Hutchison, J. E., Living Nanocrystals. Chemistry of Materials 2017, 29 (13), 5415-5425.

27. Crockett, B. M.; Jansons, A. W.; Koskela, K. M.; Johnson, D. W.; Hutchison, J. E., Radial Dopant Placement for Tuning Plasmonic Properties in Metal Oxide Nanocrystals. ACS Nano 2017, 11 (8), 7719-7728.

28. Jansons, A. W.; Koskela, K. M.; Crockett, B. M.; Hutchison, J. E., Transition Metal-Doped Metal Oxide Nanocrystals: Efficient Substitutional Doping through a Continuous Growth Process. Chemistry of Materials 2017, 29 (19), 8167-8176.

29. Cooper, S. R.; Plummer, L. K.; Cosby, A. G.; Lenox, P.; Jander, A.; Dhagat, P.; Hutchison, J. E., Insights into the Magnetic Properties of Sub-10 nm Iron Oxide Nanocrystals through the Use of a Continuous Growth Synthesis. Chemistry of Materials 2018, 30 (17), 6053-6062.

30. Bahnemann, D. W.; Kormann, C.; Hoffmann, M. R., Preparation and characterization of quantum size zinc oxide: a detailed spectroscopic study. The Journal of Physical Chemistry 1987, 91 (14), 3789-3798.

31. Spanhel, L.; Anderson, M. A., Semiconductor clusters in the sol-gel process: quantized aggregation, gelation, and crystal growth in concentrated zinc oxide colloids. Journal of the American Chemical Society 1991, $113(8), 2826-2833$.

32. Schwartz, D. A.; Norberg, N. S.; Nguyen, Q. P.; Parker, J. M.; Gamelin, D. R., Magnetic Quantum Dots: Synthesis, Spectroscopy, and Magnetism of Co2+- and Ni2+-Doped ZnO Nanocrystals. Journal of the American Chemical Society 2003, 125 (43), 13205-13218.

33. Ludi, B.; Niederberger, M., Zinc oxide nanoparticles: chemical mechanisms and classical and non-classical crystallization. Dalton Transactions 2013, 42 (35), 12554-12568.

34. Famengo, A.; Anantharaman, S.; Ischia, G.; Causin, V.; Natile, M. M.; Maccato, C.; Tondello, E.; Bertagnolli, H.; Gross, S., Facile and Reproducible Synthesis of Nanostructured Colloidal ZnO Nanoparticles from Zinc Acetylacetonate: Effect of Experimental Parameters and Mechanistic Investigations. European Journal of Inorganic Chemistry 2009, 2009 (33), 5017-5028.

35. Joo, J.; Kwon, S. G.; Yu, J. H.; Hyeon, T., Synthesis of ZnO Nanocrystals with Cone, Hexagonal Cone, and Rod Shapes via Non-Hydrolytic Ester Elimination Sol-Gel Reactions. Advanced Materials 2005, 17 (15), 1873-1877. 
36. Yang, Y.; Jin, Y.; He, H.; Wang, Q.; Tu, Y.; Lu, H.; Ye, Z., Dopant-Induced Shape Evolution of Colloidal Nanocrystals: The Case of Zinc Oxide. Journal of the American Chemical Society 2010, 132 (38), 13381-13394.

37. Bilecka, I.; Luo, L.; Djerdj, I.; Rossell, M. D.; Jagodič, M.; Jagličić, Z.; Masubuchi, Y.; Kikkawa, S.; Niederberger, M., Microwave-Assisted Nonaqueous Sol-Gel Chemistry for Highly Concentrated ZnO-Based Magnetic Semiconductor Nanocrystals. The Journal of Physical Chemistry C 2011, 115 (5), 1484-1495.

38. Meyers, S. T.; Anderson, J. T.; Hung, C. M.; Thompson, J.; Wager, J. F.; Keszler, D. A., Aqueous Inorganic Inks for Low-Temperature Fabrication of ZnO TFTs. Journal of the American Chemical Society 2008, 130 (51), 17603-17609.

39. Znaidi, L.; Soler Illia, G. J. A. A.; Benyahia, S.; Sanchez, C.; Kanaev, A. V., Oriented ZnO thin films synthesis by sol-gel process for laser application. Thin Solid Films 2003, 428 (1), 257-262.

40. LaMer, V. K.; Dinegar, R. H., Theory, Production and Mechanism of Formation of Monodispersed Hydrosols. Journal of the American Chemical Society 1950, 72 (11), 4847-4854.

41. Han, X.; Zhou, X.; Jiang, Y.; Xie, Z., The preparation of spiral ZnO nanostructures by topdown wet-chemical etching and their related properties. Journal of Materials Chemistry 2012, 22 (21), 10924-10928.

42. Kim, T.; Cho, E.-J.; Chae, Y.; Kim, M.; Oh, A.; Jin, J.; Lee, E.-S.; Baik, H.; Haam, S.; Suh, J.S.; Huh, Y.-M.; Lee, K., Urchin-Shaped Manganese Oxide Nanoparticles as pH-Responsive Activatable T1 Contrast Agents for Magnetic Resonance Imaging. Angewandte Chemie International Edition 2011, 50 (45), 10589-10593.

43. Hofmann, C.; Rusakova, I.; Ould-Ely, T.; Prieto-Centurión, D.; Hartman, K. B.; Kelly, A. T.; Lüttge, A.; Whitmire, K. H., Shape control of new FexO-Fe3O4and Fe1-yMnyO-Fe3-zMnzO4 nanostructures. Advanced Functional Materials 2008, 18 (11), 1661-1667.

44. Zhou, D.; Wang, P.; Roy, C. R.; Barnes, M. D.; Kittilstved, K. R., Direct Evidence of Surface Charges in n-Type Al-Doped ZnO. The Journal of Physical Chemistry C 2018, 122 (32), 18596-18602. 45. Wang, M.; Zhou, Y.; Zhang, Y.; Kim, E. J.; Hahn, S. H.; Seong, S. G., Near-infrared photoluminescence from ZnO. Applied Physics Letters 2012, 100 (10), 101906.

46. Janotti, A.; Walle, C. G. V. d., Oxygen vacancies in ZnO. Applied Physics Letters 2005, 87 (12), 122102.

47. Morfa, A. J.; Beane, G.; Mashford, B.; Singh, B.; Della Gaspera, E.; Martucci, A.; Mulvaney, P., Fabrication of ZnO Thin Films from Nanocrystal Inks. The Journal of Physical Chemistry C 2010, 114 (46), 19815-19821.

48. Zhu, W.; Kitamura, S.; Boffelli, M.; Marin, E.; Gaspera, E. D.; Sturaro, M.; Martucci, A.; Pezzotti, G., Analysis of defect luminescence in Ga-doped ZnO nanoparticles. Physical Chemistry Chemical Physics 2016, 18 (14), 9586-9593.

49. Wood, A.; Giersig, M.; Hilgendorff, M.; Vilas-Campos, A.; Liz-Marzán, L. M.; Mulvaney, P., Size Effects in ZnO: The Cluster to Quantum Dot Transition. Australian Journal of Chemistry 2003, 56 (10), 1051-1057.

50. Morfa, A. J.; Kirkwood, N.; Karg, M.; Singh, T. B.; Mulvaney, P., Effect of Defects on the Behavior of ZnO Nanoparticle FETs. The Journal of Physical Chemistry C 2011, 115 (16), 8312-8315.

51. Ning, J.; Liu, J.; Levi-Kalisman, Y.; Frenkel, A. I.; Banin, U., Controlling Anisotropic Growth of Colloidal ZnSe Nanostructures. Journal of the American Chemical Society 2018, 140 (44), 1462714637.

52. Jansons, A. W.; Hutchison, J. E., Continuous Growth of Metal Oxide Nanocrystals: Enhanced Control of Nanocrystal Size and Radial Dopant Distribution. ACS Nano 2016, 10 (7), 6942-6951.

53. Lounis, S. D.; Runnerstrom, E. L.; Bergerud, A.; Nordlund, D.; Milliron, D. J., Influence of Dopant Distribution on the Plasmonic Properties of Indium Tin Oxide Nanocrystals. Journal of the American Chemical Society 2014, 136 (19), 7110-7116. 
54. Staller, C. M.; Robinson, Z. L.; Agrawal, A.; Gibbs, S. L.; Greenberg, B. L.; Lounis, S. D.; Kortshagen, U. R.; Milliron, D. J., Tuning Nanocrystal Surface Depletion by Controlling Dopant Distribution as a Route Toward Enhanced Film Conductivity. Nano Letters 2018, 18 (5), 2870-2878. 55. Della Gaspera, E.; Bersani, M.; Cittadini, M.; Guglielmi, M.; Pagani, D.; Noriega, R.; Mehra, S.; Salleo, A.; Martucci, A., Low-Temperature Processed Ga-Doped ZnO Coatings from Colloidal Inks. Journal of the American Chemical Society 2013, 135 (9), 3439-3448.

56. Liang, X.; Ren, Y.; Bai, S.; Zhang, N.; Dai, X.; Wang, X.; He, H.; Jin, C.; Ye, Z.; Chen, Q.; Chen, L.; Wang, J.; Jin, Y., Colloidal Indium-Doped Zinc Oxide Nanocrystals with Tunable Work Function: Rational Synthesis and Optoelectronic Applications. Chemistry of Materials 2014, 26 (17), 5169-5178.

57. Ghosh, S.; Saha, M.; De, S. K., Tunable surface plasmon resonance and enhanced electrical conductivity of In doped ZnO colloidal nanocrystals. Nanoscale 2014, 6 (12), 7039-7051.

58. Greenberg, B. L.; Ganguly, S.; Held, J. T.; Kramer, N. J.; Mkhoyan, K. A.; Aydil, E. S.; Kortshagen, U. R., Nonequilibrium-Plasma-Synthesized ZnO Nanocrystals with Plasmon Resonance Tunable via Al Doping and Quantum Confinement. Nano Letters 2015, 15 (12), 8162-8169.

59. Shannon, R. D., Revised effective ionic radii and systematic studies of interatomic distances in halides and chalcogenides. Acta Crystallographica 1976, 32 (5), 751-767.

60. Della Gaspera, E.; Chesman, A. S. R.; van Embden, J.; Jasieniak, J. J., Non-injection Synthesis of Doped Zinc Oxide Plasmonic Nanocrystals. ACS Nano 2014, 8 (9), 9154-9163.

61. Della Gaspera, E.; Duffy, N. W.; van Embden, J.; Waddington, L.; Bourgeois, L.; Jasieniak, J. J.; Chesman, A. S. R., Plasmonic Ge-doped ZnO nanocrystals. Chemical Communications 2015, 51 (62), 12369-12372.

62. Lee, J.; Lee, S.; Li, G.; Petruska, M. A.; Paine, D. C.; Sun, S., A Facile Solution-Phase Approach to Transparent and Conducting ITO Nanocrystal Assemblies. Journal of the American Chemical Society 2012, 134 (32), 13410-13414.

63. Manthiram, K.; Alivisatos, A. P., Tunable Localized Surface Plasmon Resonances in Tungsten Oxide Nanocrystals. Journal of the American Chemical Society 2012, 134 (9), 3995-3998.

64. Nütz, T.; Felde, U. z.; Haase, M., Wet-chemical synthesis of doped nanoparticles: Bluecolored colloids of $\mathrm{n}$-doped SnO2:Sb. The Journal of Chemical Physics 1999, 110 (24), 12142-12150. 65. Buonsanti, R.; Llordes, A.; Aloni, S.; Helms, B. A.; Milliron, D. J., Tunable Infrared Absorption and Visible Transparency of Colloidal Aluminum-Doped Zinc Oxide Nanocrystals. Nano Letters 2011, 11 (11), 4706-4710.

66. Johns, R. W.; Bechtel, H. A.; Runnerstrom, E. L.; Agrawal, A.; Lounis, S. D.; Milliron, D. J., Direct observation of narrow mid-infrared plasmon linewidths of single metal oxide nanocrystals. Nature Communications 2016, 7, 11583.

67. Link, S.; Mohamed, M. B.; El-Sayed, M. A., Simulation of the Optical Absorption Spectra of Gold Nanorods as a Function of Their Aspect Ratio and the Effect of the Medium Dielectric Constant. The Journal of Physical Chemistry B 1999, 103 (16), 3073-3077. 